\section{P71 (continued)}

Objective: To improve dietary behavior of guardians of adolescents participating in a lifestyle weight management program.

Target Audience: Guardians of overweight and obese Hispanic adolescents participating in the Confidence, Ownership, Responsibility, and Exercise (CORE) lifestyle weight management program.

Theory, Prior Research, Rationale: The CORE lifestyle program was designed to improve body composition and weight management behaviors among overweight and obese Hispanic adolescents. As research suggests that effective obesity treatment in adolescents requires involvement of the family, an educational program was developed to teach guardians of CORE participants about nutrition for health promotion and weight management and improve dietary behavior. Curriculum was based on Social Cognitive Theory, the Dietary Guidelines for Americans, and American Academy of Pediatrics and Academy of Nutrition and Dietetics position papers on pediatric weight management.

Description: Nutrition education consisted of one, 60minute session per week over 12 weeks. Lessons were taught in both Spanish and English by a registered dietitian or trained nutrition intern and volunteer translator. All sessions were interactive and included hands on activities. Topics included MyPlate, sugary beverages, choosing healthy fats, and reading nutrition labels.

Evaluation: Following the program, guardians completed a retrospective, researcher-designed questionnaire to assess likelihood of engaging in healthy dietary behaviors before and after the program. Wilcoxon signed-rank tests were used to compare pre and post data. Twenty-six guardians provided data (mean age $38.8 \pm 5.7 \mathrm{y}, 70.4 \%$ female, $59.2 \% \leq$ middle-school education). Mean \pm SD attendance was $56.7 \pm 0.3 \%$ of sessions. Guardians reported increased likelihood of choosing water instead of sugary drinks, using nutrition labels, choosing whole grains, choosing vegetables in place of higher calorie foods, and choosing low fat/fat free milk and dairy (water: $P<.05$, all other behaviors: $P<.01)$.

Conclusions and Implications: The CORE nutrition education program was successful in increasing likelihood of engaging in healthy dietary behaviors among guardians of overweight and obese Hispanic adolescents. Future studies should address barriers to attendance in this population.

Funding: Jackson EMC Foundation, Mulherin Foundation.

\section{P72 Investigating Dietary and Physical Activity Patterns Among Hmong American Youth: A Cultural Perspective}

Urvashi Mulasi,PhD, MS, RDN, mulasi@csus.edu, California State University, Sacramento, 6000 J Street, Sacramento, CA 95819; Lisa Franzen-Castle, PhD, MS, RDN, University of Nebraska-Lincoln; Mical Kay Shilts, PhD, California State University, Sacramento
Background (Background, Rationale, Prior Research, and/or Theory): While obesity and type-2 diabetes rates have risen among Hmong, limited research has investigated their nutritional status. It is important to investigate Hmong dietary and physical activity patterns so that timely nutritional interventions may be planned. Objective: We investigated the factors that influence dietary and physical activity patterns among Hmong American youth (14-25-year-olds) in the Sacramento region.

Study Design, Setting, Participants, Intervention: Eight focus groups $(n=44)$ were conducted. Each focus group lasted for 60-90 minutes with 4 to 7 youth per group. Participants completed a brief questionnaire regarding smoking, alcohol, and dietary and physical activity behaviors adapted from the Centers for Disease Control and Prevention's Youth Risk Surveillance Survey. Focus groups were audio taped and transcribed verbatim; transcriptions were analyzed for themes and discrepancies were noted and resolved.

Outcome Measures and Analysis: Descriptive data were analyzed using IBM SPSS Statistics software (version 24). Significance level was set at $P<.05$.

Results: Twenty-nine female and 15 male youth participated in the study, mean $( \pm S D)$ age was $19 \pm 3$ years. Most youth $(84 \%)$ were born in the United States, seven (16\%) were born in either Laos or Thailand. Questionnaire results indicated that $26 \%$ of youth reported eating fried foods 4-6 times during the past week. Furthermore, 51\% did not eat green salad and 14\% did not consume any fruit during the past week. Focus group data suggested that preference for culturally familiar foods made youth's intake of vegetables challenging. While parental influences negatively influenced female youth's physical activity patterns, cultural expectations to fit in appeared to affect male youth's alcohol consumption.

Conclusions and Implications: Cultural factors affected the youths' health and well-being. Future nutrition intervention among Hmong youth is recommended to be culturally-specific with an understanding of parental influences and/or culturally-driven barriers to healthy living. More data with a larger sample size is needed to gain an understanding about Hmong health in this region.

Funding: Probationary Faculty Development Grant Program at the California State University, Sacramento.

\section{P73 Involving Students and Increasing Communication: Results of a Behavioral Economics Informed Lunchroom Intervention in Middle Schools}

Grace Ryan, MPH, grace-ryan@uiowa.edu, University of Iowa, College of Public Health, N475 College of Public Health Building, 145 N Riverside Drive, Iowa City, IA 52246; Patrick J. Brady, MS; Natoshia M. Askelson, PhD, MPH; Patti Delger, LDN, RD, Iowa Department of Education; Carrie Scheidel, MPH

Objective: The objective of this project was to implement an intervention informed by behavioral economics

Continued on page $S 42$ 


\section{P73 (continued)}

principles to increase healthy choices made by students during lunch. This was accomplished by involving students in intervention activities and encouraging communication between students and food service staff (FSS) through communication training and planned interactions.

Target Audience: Students, food services directors, and FSS from six middle schools participated in this intervention.

Theory, Prior Research, Rationale: This project was based on behavioral economics and grounded in prior work conducted with high school students.

Description: This intervention consisted of two components: changes to the lunchroom and communication training for FSS. Students worked with food service staff throughout the year to identify and implement changes to their lunchroom. Training was provided to FSS through webinars, and visual materials were distributed that encouraged positive communication with students at the service line.

Evaluation: The evaluation of this project included a lunchroom assessment tool, online surveys with parents, students, and staff, food production records, and indepth interviews with food service directors. Assessment tool scores, online surveys, the number of servings of fruits, vegetables, and milk varieties were compared pre and post intervention. Interviews were audio recorded, transcribed, and coded for relevant themes.

Conclusions and Implications: The intervention implemented low/no cost changes and encouraged positive communication between FSS and students to promote healthy food choices. Five schools improved their scores on the assessment tool. Food production record data indicated that servings of fruit $(n=2)$, vegetables $(n=3)$, and milk $(n=4)$ increased following the intervention. Response to the project from food service directors was positive, as they noted improved communication and relationships with their students as a result of the project. No changes were seen in comparison of surveys that indicated an intervention effect. Lunchroom interventions should include students to identify changes and more importantly focus on increasing student interaction with staff. Funding: USDA Team Nutrition.

\section{P74 Key Informants' Perceptions of Needs Among Nevada's Supplemental Nutrition Assistance Program Education (SNAP-Ed) Target Audience}

Megan Wahrenburg, MS, mwahrenburg@cabnr.unr.edu, University of Nevada, Reno, 1664 N Virginia Street, M.S. 202, Reno, NV 89557; Jamie Benedict, PhD, LD, RDN; Heather Kerwin, MPH

Background (Background, Rationale, Prior Research, and/or Theory): Interviews with key informants throughout Nevada were conducted for the purpose of describing the most pressing nutrition and physical activity education needs of the SNAP-Ed target audience and examining relevant community characteristics and other environmental factors that shape behavior. The findings will complement other assessment data and be used to guide SNAP-Ed programming in the future.

Objective: Describe key informants' perceptions regarding the nutrition and physical activity education needs of SNAP participants and others residing in low-income communities in Nevada.

Study Design, Setting, Participants, Intervention: Semi-structured interviews were conducted with professionals $(\mathrm{n}=35)$ who had knowledge of SNAP households and others residing in low-income communities. Participants were strategically selected to include those from a variety of Nevada geographies and those with expertise in various disciplines, many of which had daily contact with members of the target audience. Interviews were conducted by phone and audio-recorded.

Outcome Measures and Analysis: Qualitative data on nutrition and physical activity education needs and potential policy, systems, and environmental (PSE) approaches to stimulate behavior change was analyzed using the grounded theory approach.

Results: Key informants indicated that education on healthful shopping, food resource management, and cooking is necessary to achieve healthful diets. However, they noted that environmental barriers often make it difficult for Nevadans to make healthful choices. Possible PSE approaches included improved access to healthy food and opportunities for physical activity, incentives for healthy behavior, nutrition signage in grocery stores, and increased collaboration among community partners. In general, the informants voiced optimism and enthusiasm for moving forward as reflected in the following theme that emerged from the interviews, "Let's make the healthy choice the easy choice-we can do it!"

Conclusions and Implications: This study provided new information that can inform SNAP-Ed programming, including potential methods to make it easier for SNAP households and others residing in low-income communities to choose healthful foods and be physically active more often.

Funding: Supplemental Nutrition Assistance Program-Education.

\section{P75 Long-Term Impact of the Child Health Assessment in the Pacific (CHAP) Undergraduate Summer Fellowship Program}

Marie K. Fialkowski,PhD, RD, mariekf@hawaii.edu, University of Hawaii, Manoa, 1955 East West Road, AgSci 216, Honolulu, HI 96822; M'Randa Sandlin, PhD; Rachel Novotny, PhD, RD; Margaret Hattori-Uchima, PhD, $R N$, University of Guam; Patricia Coleman, BS, Northern Marianas College; Chrystie Naeole, PhD, Chaminade University

Objective: To evaluate the long-term impact of the Child Health Assessment in the Pacific Undergraduate Summer Fellowship Program (CHAP) on alumni educational and career goals.

Continued on page $S 43$ 